\title{
The Relation between the Frequency Distributions of Sieve Diameters and Fall Velocities of Sediment Particles
}

\author{
John F. KenNedy ${ }^{1}$ and RoberT C. Y. KoH \\ W. M. Keck Laboratory of Hydraulics and Water Resources \\ California Institute of Technology, Pasadena
}

\begin{abstract}
A relation between the frequency distributions of sieve diameters and fall velocities is developed. The relation shows that if the sieve diameters of a sediment are log-normally distributed, the fall velocities will also have this distribution, but with a different standard deviation which can be calculated. The results of detailed measurements of the fall velocities of two different natural sands are presented, and the distributions of fall velocities are found to be in good agreement with the predicted distributions. The sedimentation diameters determined from the measured fall velocities agree very well with those predicted from the sieve diameters by the Inter-Agency Committee on Water Resources [1957].
\end{abstract}

\section{INTRODUCTION}

In problems involving the transport of solids by a fluid, or the separation of solids from a transporting fluid, the most important single property of the sediment in determining its transport properties and effect on the fluid flow is the fall velocity of the sediment particles in the transporting fluid. The fall velocity of a particle is a complex function of the size, shape, and density of the particle, the properties of the fluid, the characteristics of the flow, and the concentration and distribution of other particles surrounding the particle in question. This in situ fall velocity usually cannot be determined; instead, the terminal fall velocities of representative individual particles falling under gravity in the transporting fluid, at rest and effectively unbounded, are used to describe the settling properties of the sediment. When the fall velocity of a particle is known, it can conveniently be described by the sedimentation diameter of the particle, $D_{u}$, which is defined as the diameter of a sphere, of the same density as the particle, which has the same fall velocity in the same quiescent fluid at the same temperature. Conversely, if the sedimentation diameter of a particle is known, its fall velocity can be computed from data on the fall velocities of spheres.

Natural sediments, such as sand, are composed of particles of many sizes and shapes, and con-

1 Now at Hydrodynamics Laboratory, Department of Civil and Sanitary Engineering, Massachusetts Institute of Technology, Cambridge. sequently the frequency distributions of fall velocities and sedimentation diameters must be considered. The determination of the frequency distribution of the fall velocities of a sediment by measuring the fall velocities of individual particles of a sample of the sediment is quite tedious. Instead, a sieve analysis is usually used to determine the distribution of the sieve diameters of the particles. The sieve diameter of a particle, $D^{\prime}$, is usually defined as the geometric mean of the openings of the last sieve through which the particle passed and the sieve on which it is retained; this is the definition that will be used here. To describe the fall velocity characteristics of a sediment, it is necessary to know the relation between the sieve diameters and the sedimentation diameters of the particles, and between the frequency distribution of sedimentation diameters and the frequency distribution of fall velocities.

The main objective of this paper is to develop a relation between the frequency distributions of sieve diameters and fall velocities. In this connection, some analytic considerations of the fall velocities of spheres outside the Stokes range are presented. The second objective is to evaluate a method which has been presented [Inter-Agency Committee on Water Resources, 1957] for determining sedimentation diameters from sieve diameters. The results of some careful measurements of fall velocities of two different sands are given, and these data are used to evaluate the relations derived for predicting the frequency distribution of fall velocities. The sedimentation 
diameters obtained from the measurements are compared with those predicted by the InterAgency report. This work was done in connection with a recent investigation at California Institute of Technology on the mechanics of sediment transportation [Kennedy, 1961]. The sands used in this study of fall velocities were also used in the sedimentation study.

\section{Fall Velocities of Spheres}

By equating the submerged weight of a sphere to the drag force acting on it, we can express its terminal fall velocity as

$$
w^{2}=\frac{4}{3}(s-1) \frac{g D}{C_{D}}
$$

where

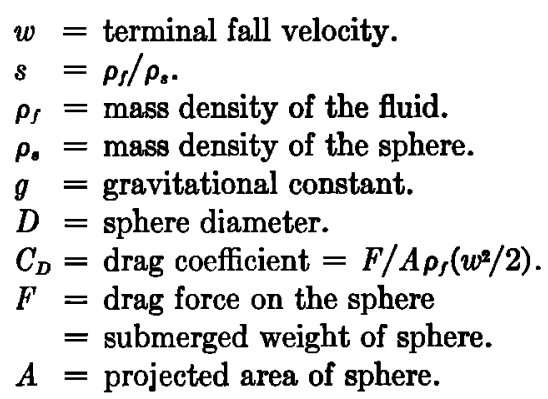

The drag coefficient outside the Stokes range, which ends at a Reynolds number of about 0.4, must be determined experimentally and is usually expressed as a function of the Reynolds number. Graphs giving this relation, such as the one shown in Figure 1 [Rouse, 1950, p. 122], can be found in most textbooks on fluid mechanics. However, such graphs are not particularly convenient for the determination of fall velocity because a trial and error procedure is required. To make a direct determination possible, Rouse $[1950$, p. 781] has presented the drag coefficientReynolds number relation for quartz spheres in water and air as fall velocity plotted against sphere diameter with temperature as a third variable, as shown in Figure 2. This graph makes the determination of fall velocity quite convenient but does not give a closed form relation which can be used in mathematical analyses of fall velocity problems. Such a relation will now be developed.

Since $C_{D}$ is a function of only the Reynolds number, (1) gives $w$ as a function of $D, s$, and the kinematic viscosity $\nu$. Therefore, $d w=\frac{\partial w}{\partial D} d D+\frac{\partial w}{\partial \nu} d \nu+\frac{\partial w}{\partial(s-1)} d(s-1)$

The following partial derivatives can be obtained from (1):

$$
\begin{gathered}
2 w \frac{\partial w}{\partial D}=\frac{4}{3}(s-1)\left[\frac{g}{C_{D}}-\frac{g D}{C_{D}^{2}} \frac{d C_{D}}{d\left(\log C_{D}\right)}\right. \\
\left.\cdot \frac{d\left(\log C_{D}\right)}{d(\log R)} \frac{d(\log R)}{d R} \frac{\partial R}{\partial D}\right] \\
2 w \frac{\partial w}{\partial \nu}=\frac{4}{3}(s-1)\left[-\frac{g D}{C_{D}{ }^{2}} \frac{d C_{D}}{d\left(\log C_{D}\right)}\right. \\
\left.\cdot \frac{d\left(\log C_{D}\right)}{d(\log R)} \frac{d(\log R)}{d R} \frac{\partial R}{\partial \nu}\right] \\
2 w \frac{\partial w}{\partial(s-1)}=\frac{4}{3} g D\left[\frac{1}{C_{D}}-\frac{(s-1)}{C_{D}{ }^{2}} \frac{d C_{D}}{d\left(\log C_{D}\right)}\right. \\
\left.\cdot \frac{d\left(\log C_{D}\right)}{d(\log R)} \frac{d(\log R)}{d R} \frac{\partial R}{\partial w} \frac{\partial w}{\partial(s-1)}\right]
\end{gathered}
$$

where $R$ is the Reynolds number, $R=w D / \nu$. The quantity $\left(d\left(\log C_{D}\right) /(d(\log R))\right.$ is the slope of the $C_{D}-R$ curve on a logarithmic plot, and its negative will be denoted by $m$.

$$
m=-\frac{d\left(\log C_{D}\right)}{d(\log R)}
$$

Substituting (1) and (6) into (3), (4), and (5) and carrying out the indicated differentiation, we obtain

$$
\begin{aligned}
& \frac{\partial w}{\partial D}=\frac{1+m}{2-m} \frac{w}{D} \\
& \frac{\partial w}{\partial \nu}=\frac{-m}{2-m} \frac{w}{\nu}
\end{aligned}
$$

and

$$
\frac{\partial w}{\partial(s-1)}=\frac{1}{2-m} \frac{w}{s-1}
$$

Substituting (7), (8), and (9) into (2) yields

$$
\begin{aligned}
\frac{d w}{w}=\frac{1+m}{2-m} \frac{d D}{D} & -\frac{m}{2-m} \frac{d \nu}{\nu} \\
& +\frac{1}{2-m} \frac{d(s-1)}{s-1}
\end{aligned}
$$

Values of $m,(1+m) /(2-m), m /(2-m)$, and $1 /(2-m)$ are tabulated with Reynolds 


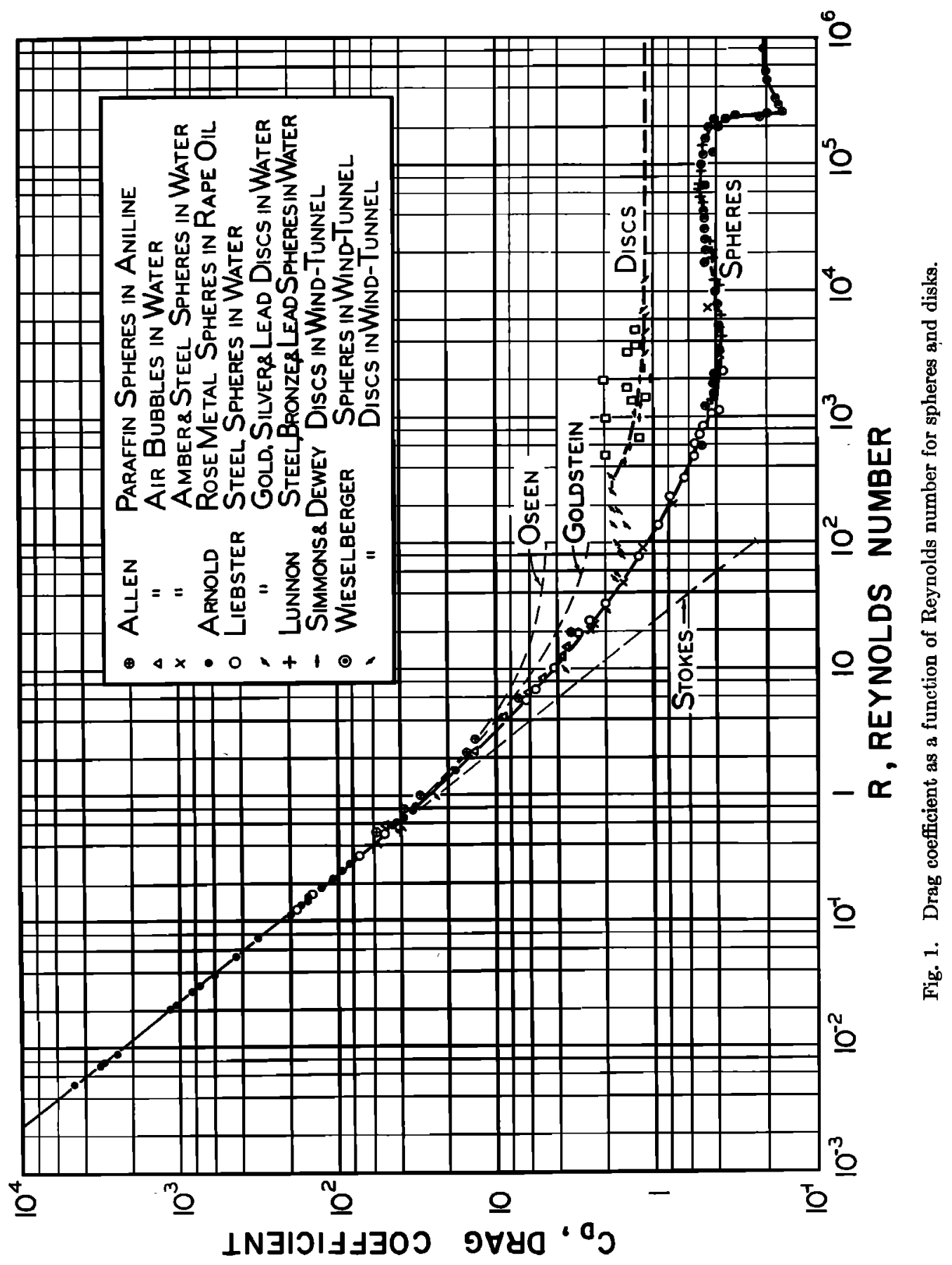




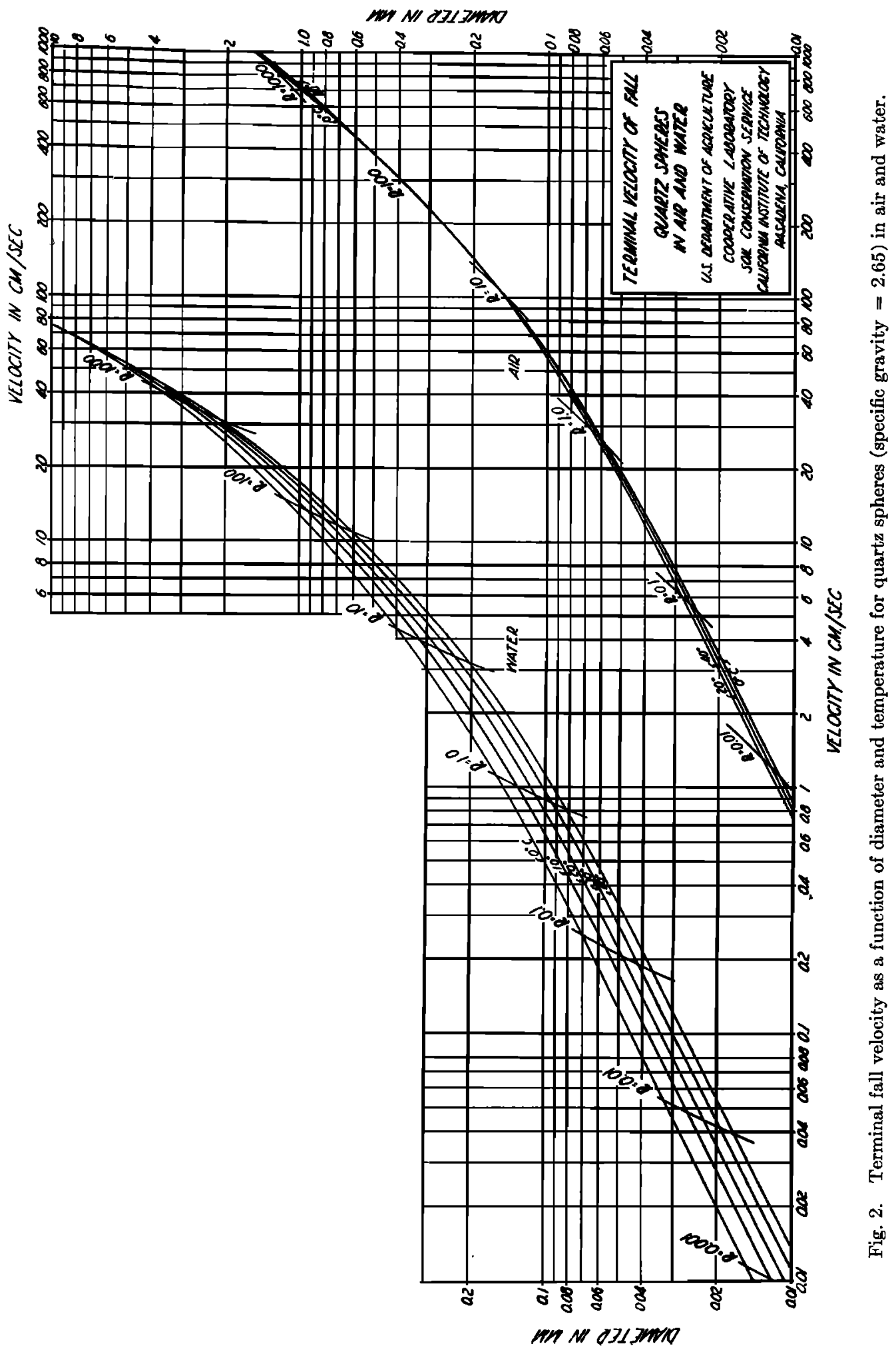


number in Table 1 and are shown plotted against Reynolds number in Figure 3.

As can be seen in Figure 3, the relation between the Reynolds number and the three parameters appearing in (10) can be closely approximated by

$$
\frac{1+m}{2-m}=1.70 R^{-0.120}
$$

for Reynolds numbers between 1 and 1000;

$$
\frac{m}{2-m}=0.88 R^{-0.220}
$$

for $R$ between 2 and 400 ; and

$$
\frac{1}{2-m}=0.90 R^{-0.065}
$$

for $R$ between 1 and 5000. Substitution of (11), (12), and (13), into (10) yields

$$
\begin{array}{r}
\frac{d w}{w}=1.70 R^{-0.120} \frac{d D}{D}-0.88 R^{-0.220} \frac{d \nu}{\nu} \\
+0.90 R^{-0.065} \frac{d(s-1)}{s-1}
\end{array}
$$

valid for Reynolds numbers between 2 and 400 .

Equation 14 is nonlinear and cannot easily be integrated in closed form. However, the relation between $w$ and $D$ can be obtained for fixed values of $\nu$ and $s$. In this case, $d \nu=d(s-$ $1)=0$, and (10) reduces to

$$
\frac{d w}{w}=\frac{1+m}{2-m} \frac{d D}{D}
$$

and (14) becomes

$$
d w / w=1.70 R^{-0.120} d D / D
$$

Substituting for $R$ and carrying out the integration yields

$$
w^{0.120}=-1.70 \nu^{0.120} D^{-0.120}+C
$$

where $C$ is an integration constant that depends on the density and viscosity of the fluid and the density of the spheres. For quartz spheres in water at $25^{\circ} \mathrm{C}\left(s=2.65, \nu=0.966 \times 10^{-6}\right.$ $\left.\mathrm{ft}^{2} / \mathrm{sec}\right), C=1.76$, and

\begin{tabular}{|c|c|c|c|c|}
\hline \multirow{2}{*}{\begin{tabular}{l}
\multicolumn{1}{c}{$R$} \\
Reynolds \\
Number
\end{tabular}} & \multirow{2}{*}{$-\frac{\begin{array}{c}m \\
d\left(\log C_{D}\right)\end{array}}{d(\log R)}$} & $1+m$ & $m$ & 1 \\
\hline & & $2-m$ & $2-m$ & $2-m$ \\
\hline \multicolumn{5}{|l|}{0.4 and } \\
\hline less & 1.000 & 2.000 & 1.000 & 1.000 \\
\hline 1 & 0.890 & 1.703 & 0.802 & 0.901 \\
\hline 2 & 0.835 & 1.575 & 0.717 & 0.858 \\
\hline 4 & 0.770 & 1.439 & 0.626 & 0.813 \\
\hline 10 & 0.685 & 1.281 & 0.521 & 0.760 \\
\hline 20 & 0.635 & 1.197 & 0.465 & 0.733 \\
\hline 40 & 0.570 & 1.098 & 0.399 & 0.699 \\
\hline 100 & 0.475 & 0.967 & 0.311 & 0.656 \\
\hline 200 & 0.417 & 0.895 & 0.263 & 0.632 \\
\hline 400 & 0.365 & 0.835 & 0.223 & 0.612 \\
\hline 800 & 0.300 & 0.765 & 0.176 & 0.588 \\
\hline 1000 & 0.275 & 0.739 & 0.159 & 0.580 \\
\hline 2000 & 0.145 & 0.617 & 0.078 & 0.539 \\
\hline 5000 & 0 & 0.500 & 0 & 0.500 \\
\hline
\end{tabular}

$$
w=\left[1.76-0.426(1 / D)^{0.120}\right]^{1 / 0.120}
$$

for Reynolds numbers between 1 and 1000 .

Similarly, the relation between $w$ and $\nu$ for fixed $D$ and $s$ can be obtained by taking $d D=d(s-1)=0$ in (14). Then
TABLE 1. Parameters for Equation 10

$$
d w / w=-0.88 R^{-0.220} d \nu / \nu
$$

which can be integrated to yield

$$
w=\left[k-0.88(\nu / D)^{0.220}\right]^{1 / 0.220}
$$

for $R$ between 2 and 400, where $k$ is a constant that depends on $s$ and $D$.

Finally, the relation between $w$ and $s$ for fixed $D$ and $\nu$ can also be obtained from (14). Proceeding as above, we get

$w=\left[p+0.059(\nu / D)^{0.065} \ln (s-1)\right]^{1 / 0.065}$

where $p$ is a constant that depends on $\nu$ and $D$. Equation 19 is valid for $R$ between 1 and 5000 .

When these relations are applied to particles other than spheres, $D$ should be replaced by $D_{\imath}$.

Relation between the Fregdency Distribution of Sedimentation Diameters aNd THE Frequency Distribution of Fall Velocities

If the frequency distribution function of the sedimentation diameters of a sediment is known and the frequency distribution of the fall velocities is desired, the problem becomes one of determining the frequency distribution of a function of a random variable. In this case, the sedimentation diameter $D$, is the random variable whose frequency distribution is known, and (15) determines the function of $D$, for which the frequency distribution is desired. Let $f\left(D_{s}\right)$ be 


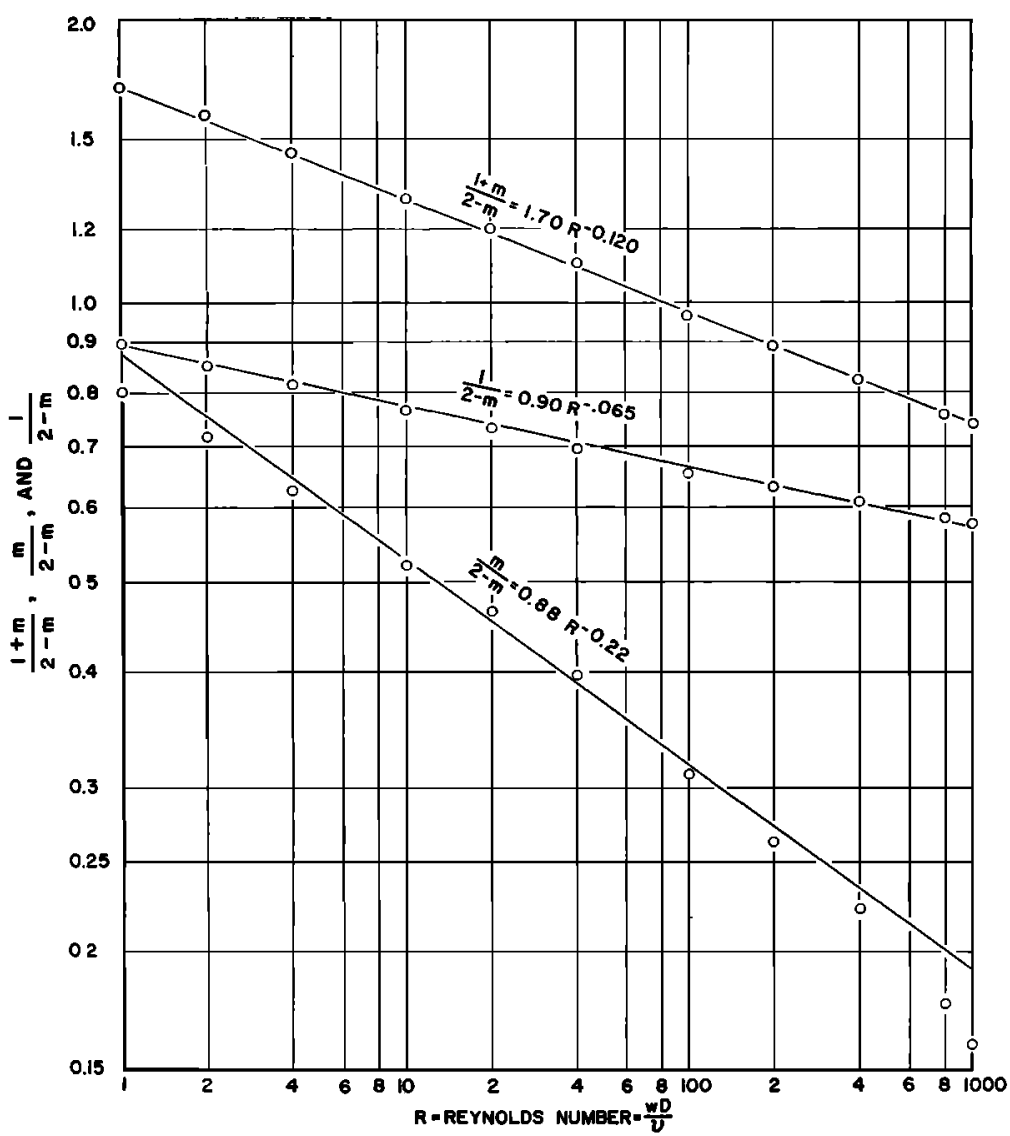

Fig. 3. Values of $(1+m) /(2-m), m /(2-m)$, and $1 /(2-m)$ as a function of Reynolds number.

the frequency distribution function of the sedimentation diameters, and let $g(w)$ be the frequency distribution function of the fall velocities. The probability that $w$ is between $w_{0}$ and $w_{0}+d w$ is the same as the probability that $D$. is between $D_{s 0}$ and $D_{s 0}+d D_{s}$, where $D_{s}=D_{s}(w)$ is the functional relation between $D_{a}$ and $w$, (15) or (16), and $D_{\iota_{0}}=D_{0}\left(w_{0}\right)$. Thus $f\left(D_{s}\right) d D_{\iota}=$ $g(w) d w$, which can be written as

$$
g(w)=f\left[D_{s}(w)\right] \frac{d D_{s}(w)}{d w}
$$

If the frequency distribution of $D$, is known or assumed, (16) can be substituted into (20) and the corresponding $g(w)$ determined.

For natural sands and also for many particulate materials such as glass and plastic beads produced by manufacturing processes, it is observed that the logarithms of the particle sieve diameters are distributed according to the normal error law.
This distribution is called the log-normal distribution. For example, the sieve diameter distributions of the sands used in this investigation are very nearly log normal except for the tails (see Fig. 5). To derive an expression for the frequency distribution of settling velocities it will be assumed that the logarithms of the sedimentation diameters are also normally distributed. Thus

$$
\begin{aligned}
f\left(\log D_{s}\right) & =\frac{1}{\sigma \sqrt{2 \pi}} \\
& \cdot \exp \left\{-\frac{1}{2}\left[\frac{\log D_{s}-\mu}{\sigma}\right]^{2}\right\}
\end{aligned}
$$

where

$\mu=$ mean of $\log D_{a}$.

$\sigma=$ standard deviation of $\log D_{\mathbf{s}}$.

The frequency distribution of fall velocities is 
obtained by solving (16) for $D_{0}$ and substituting $D$, and (21) into (20):

$$
\begin{aligned}
& g(w)=f\left[\log D_{\varepsilon}(w)\right] \frac{d \log D_{x}}{d w} \\
& =\frac{1}{\sigma \sqrt{2 \pi}} \frac{\log e}{C w^{0.880}-w} \\
& \cdot \exp \left\{-\frac{1}{2}\left[\frac{\log \nu\left(\frac{C-w^{0.120}}{1.70}\right)^{-1 / 0.120}-\mu}{\sigma}\right]^{2}\right\}
\end{aligned}
$$

Similarly,

$$
\begin{aligned}
& g(\log w)=f\left[\log D_{s}(w)\right] \frac{d \log D_{s}}{d \log w} \\
& =\frac{1}{\sigma \sqrt{2 \pi}} \frac{1}{C w^{-0.120}-1} \\
& \cdot \exp \left\{-\frac{1}{2}\left[\frac{\log \nu\left(\frac{C-w^{0.120}}{1.70}\right)^{-1 / 0.120}-\mu}{\sigma}\right]^{2}\right\}
\end{aligned}
$$

Equations 22 and 23 show that neither $w$ nor $\log w$ is exactly normally distributed, since a function of $w$ appears in the coefficients of the exponentials. However, for a sediment containing a small range of particle sizes, the logarithms of the fall velocities can be shown to be exactly normally distributed. For this case, the quantity $(1+m) /(2-m)$ is nearly constant, and (15) can be integrated to yield

$$
w=C_{1} D_{a^{(1+m) /(2-m)}}
$$

where $C_{1}$ is a dimensional constant. Use of (24) is equivalent to replacing the part of the curve of Figure 2 covered by the sediment by a straight line segment. Substitution of (21) and (24) into (20) yields where $\left(D_{s}\right)_{50}$ is the sedimentation diameter such that 50 per cent by weight of the sediment has a smaller sedimentation diameter; similarly, 15.9 per cent by weight of the sediment has a sedimentation diameter smaller than $\left(D_{s}\right)_{15.0}$. The quantity $\left(D_{s}\right)_{50} /\left(D_{s}\right)_{15.9}$ is denoted by $\sigma$, and called the geometric standard deviation of the sedimentation diameters. Similarly, $\mu=\log$ $\left(D_{0}\right)_{60}$, where $\left(D_{1}\right)_{50}$ is called the geometric mean sedimentation diameter, and is the same as the median by weight. Substituting these quantities into (25) results in

$$
\begin{aligned}
g(w) & =\frac{2-m}{1+m} \frac{1}{\log \sigma_{s} \sqrt{2 \pi}} \\
& \cdot \exp \left\{-\frac{1}{2}\left[\frac{\log w-\log w_{50}}{\frac{1+m}{2-m} \log \sigma_{s}}\right]^{2}\right\}
\end{aligned}
$$

where

$$
w_{50}=C_{1}\left[\left(D_{s}\right)_{50}\right]^{(1+m) /(2-m)}
$$

For a sediment with a range of particle sizes small enough so that (24) is valid, (27) shows that $\log w$ is also normally distributed with a geometric mean of $w_{50}$ given by (28) and a geometric standard deviation $\sigma_{w}$ of

$$
\sigma_{w}=\left(\sigma_{s}\right)^{(1+m) /(2-m)}
$$

Since $\sigma_{s}$ must always be greater than unity (or equal to unity for a uniform sediment), (29a) shows that $\sigma_{w}$ will be less than or greater than $\sigma_{s}$ according as $(1+m) /(2-m)$ is less than or greater than unity. From Figure 3, $(1+m) /(2-m)=1.0$ when $R=85$. Therefore, when the Reynolds number is less than 85 for all particles of a sediment, $\sigma_{a}$ will be less than $\sigma_{w}$, and the opposite will be true for sediments with $R$ greater than 85 for all particles.

$$
g(w)=\frac{2-m}{1+m} \frac{1}{\sigma \sqrt{2 \pi}} \exp \left\{-\frac{1}{2}\left[\frac{\log w-\frac{1+m}{2-m} \log \left(D_{s}\right)_{50}-\log C_{1}}{\frac{1+m}{2-m} \sigma}\right]\right\}
$$

Since $\sigma$ is the standard deviation of $\log D_{s}$,

$$
\begin{aligned}
\sigma & =\log \left(D_{s}\right)_{50}-\log \left(D_{s}\right)_{15.9} \\
& =\log \frac{\left(D_{s}\right)_{50}}{\left(D_{s}\right)_{15.9}}
\end{aligned}
$$

When a sediment contains significant quantities of particles with both $R$ greater than and less than 85 , no definite conclusion can be drawn, although (29) should still be qualitatively valid if the value of $(1+m) /(2-m)$ for $w_{50}$ is used. 
Usually, only the frequency distribution of the sieve diameters is known. As discussed in section 6 , if a sediment contains a limited range of particle sizes, the sieve and sedimentation diameters differ by only a nearly constant factor. Therefore, if in (24) through (29a) $D^{\prime}$ is used instead of $D_{a}$, the only difference is that a different constant coefficient must be used in (24). Since the constant does not appear in the distribution of fall velocities (equation 27), the fall velocities should be log-normally distributed if the sieve diameters have this distribution. In this case, $\sigma$, is replaced by the geometric standard deviation of the sieve diameters, $\sigma_{0}$, in (27), and $(29 a)$ becomes

$$
\sigma_{w}=\left(\sigma_{\theta}\right)^{(1+m) /(2-m)}
$$

\section{Experimental Apparatus and Procedure}

To obtain representative samples of the two sands from the laboratory sedimentation flumes to be used for the fall velocity measurements, a sample weighing several pounds was removed from five or six locations along each flume. Each of the two large samples was divided into successive halves with a Jones sample-splitter until a sample of approximately 30 grams of each sand remained. A sieve analysis was then made on each of these samples.

The sieve analyses were performed with a fourth-root-of-two series of 8-inch-diameter, half-depth Tyler laboratory sieves. The sieves were shaken 15 minutes on a Tyler Rotap sieve shaker. Each sieve fraction (sand contained between successive sieves) was weighed to the nearest $10 \mathrm{mg}$ on an analytic balance. To obtain particles of the finer sand for the fall-velocity measurements, each sieve fraction was passed through a miniature sample-splitter until a sample of approximately $\mathbf{5 0}$ particles remained. For the coarser sand, exactly 50 grains were selected at random from each sieve fraction. Each of these grains was dropped into quiescent tap water, and the time required for the grain to fall a given distance between two marks on the settling column was measured with a stop watch to the nearest tenth of a second. The distances were selected to give fall times between 6.5 and 25 seconds. The particles were released under water and were allowed several inches of fall to attain their terminal velocity before reaching the first mark, where the time measurement was begun.
Two different settling columns were used: a simple glass cylinder 3.75 inches in diameter and 66 inches long, and a liter graduate 2.38 inches in diameter and 18 inches long. The larger column was used when the required fall distance was greater than 12 inches. At intervals of about 15 minutes, the water in the column was thoroughly mixed to break up thermal gradients, and the temperature was measured with an immersion thermometer. Hot or cold water was then added as required to bring the temperature to the desired value, and the water was again mixed. Fall-velocity measurements were not resumed until the water again became quiescent. In this way, the temperature was kept within $1^{\circ} \mathrm{C}$ (in most cases within $0.5^{\circ} \mathrm{C}$ ) of the desired value. Figure 2 indicates that a temperature variation of $1^{\circ} \mathrm{C}$ results in a change of less than 2 per cent in the fall velocity of even the smallest particles used $(0.08 \mathrm{~mm})$.

It was observed that the particles often did not fall straight down, but followed an erratic spiral path. The larger particles often had complex rotating and oscillating motions also. Such rotations and oscillations were not observed with the finer particles, although they may have been present and escaped notice.

\section{Sand Characteristics}

Sieve analyses. Both of the sands used are predominantly quartz. They were obtained from a local foundry supply house and were given no further treatment except washing. Photomicrographs of the two sands are shown in Figure 4. Sand 1 is from the St. Peter formation and is marketed commercially as 'Ottawa sand.' The finer sand, sand 2, is a Nevada sand. It is somewhat more angular than sand 1.

The cumulative distributions, by weight, of the sieve diameters of the sands are shown in Figure 5, plotted on a logarithmic-probability graph as suggested by Otto [1939]. The fact that the distributions plot as straight lines, except for the tails, indicates that the logarithms of the sieve diameters do have a normal distribution; this is the distribution (21) that was assumed for the sedimentation diameters in section 3 . This distribution is completely defined when the geometric mean sieve diameter $D_{o}$ (which is the same as the median by weight) and the geometric standard deviation of the sieve diameters $\sigma_{a}$ are given. 


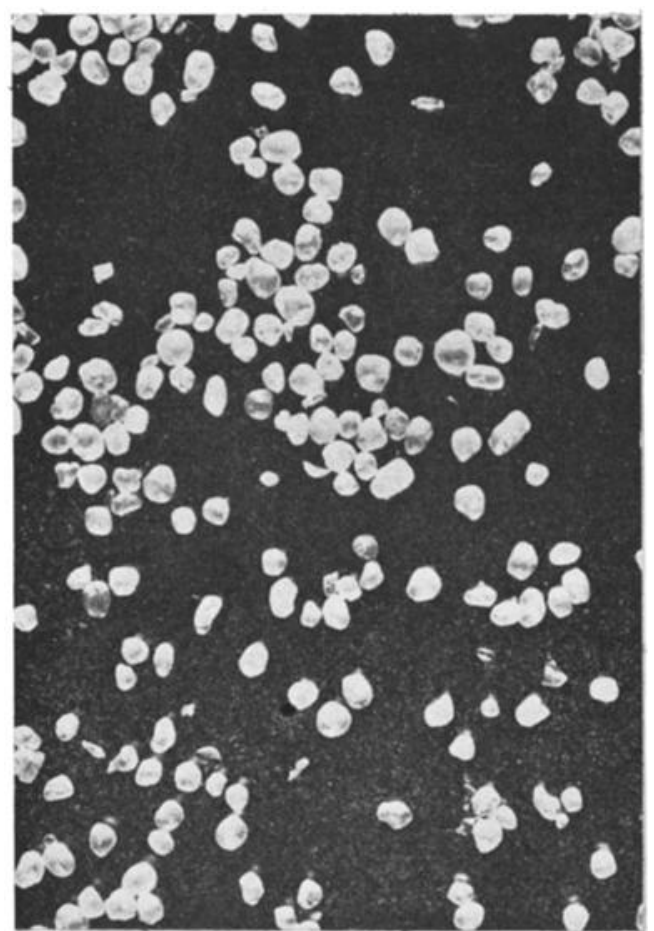

$\mathbf{a}$

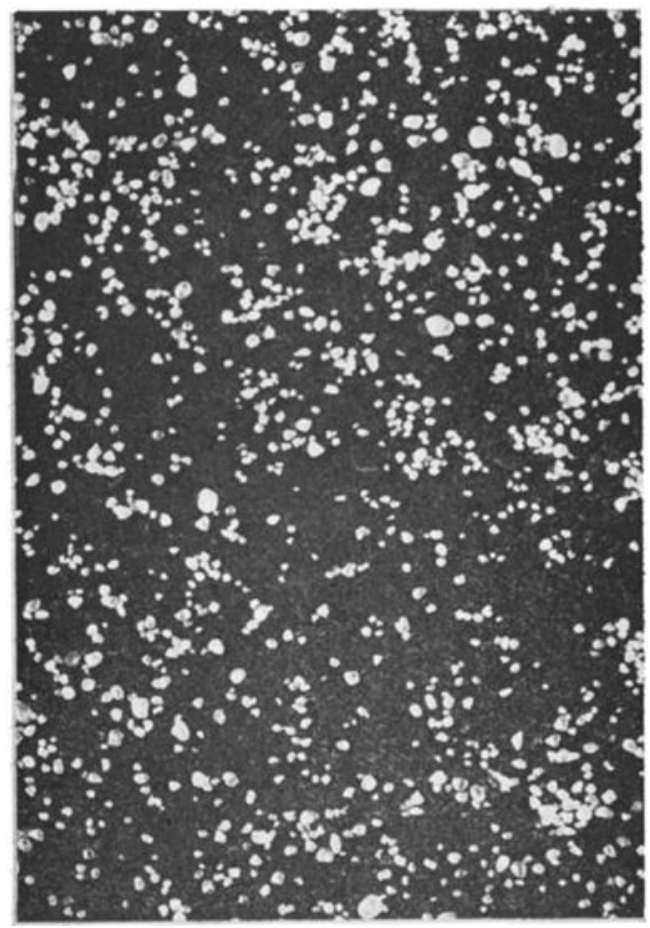

b

\section{$5 \mathrm{~mm}$}

Fig. 4. Photomicrographs of sands used.

(a) Sand 1. Geometric mean sieve diameter $=0.549 \mathrm{~mm}$.

(b) Sand 2. Geometric mean sieve diameter $=0.233 \mathrm{~mm}$.

Fall-velocity distribution. To determine the distribution of fall velocities, the number of determinations of fall velocities from each sieve fraction was first reduced to $\mathbf{5 0}$ and multiplied by a weighting factor which was the percentage, by weight, of the total sample in the sieve fraction. The number of occurrences in each velocity increment (velocity increments of 0.0050 $\mathrm{ft} / \mathrm{sec}$ were used for sand 1 and $0.0020 \mathrm{ft} / \mathrm{sec}$ for sand 2) for each sieve fraction was then counted; this gave the frequency distribution for each sieve fraction. The frequency distributions for all sieve fractions were added to obtain the frequency distribution for a representative sample consisting of $\mathbf{5 0 0 0}$ particles (50 particles in each sieve fraction $\times 100(\%)$ weight of total sample $=5000$ ). Thus, the total number. of occurrences in the ith velocity increment, $N_{i}$, is

$$
N_{i}=\sum_{m=1}^{M} \frac{50}{\gamma_{m}} n_{m i} p_{m}
$$

where

$$
\begin{aligned}
n_{m i}= & \text { number of occurrences in the ith ve- } \\
& \text { locity increment for the sand in the } \\
& m \text { th sieve fraction. } \\
p_{m}= & \text { percentage, by weight, of sample re- } \\
& \text { tained in the } m \text { th sieve fraction. } \\
M= & \text { number of sieve fractions. } \\
\gamma_{m}= & \text { number of measurements of fall velocity } \\
& \text { for the } m \text { th sieve fraction. }
\end{aligned}
$$

Note that in this procedure it is assumed that weights of all the individual particles in each sieve fraction are the same. The cumulative distribution by weight of fall velocities was then calculated as the subtotal of the number of occurrences of velocities given by the frequency distribution.

The cumulative distributions of fall velocities are shown in Figure 6, plotted on an arithmeticprobability graph, and in Figure 7, plotted on a logarithmic-probability graph. These figures show 


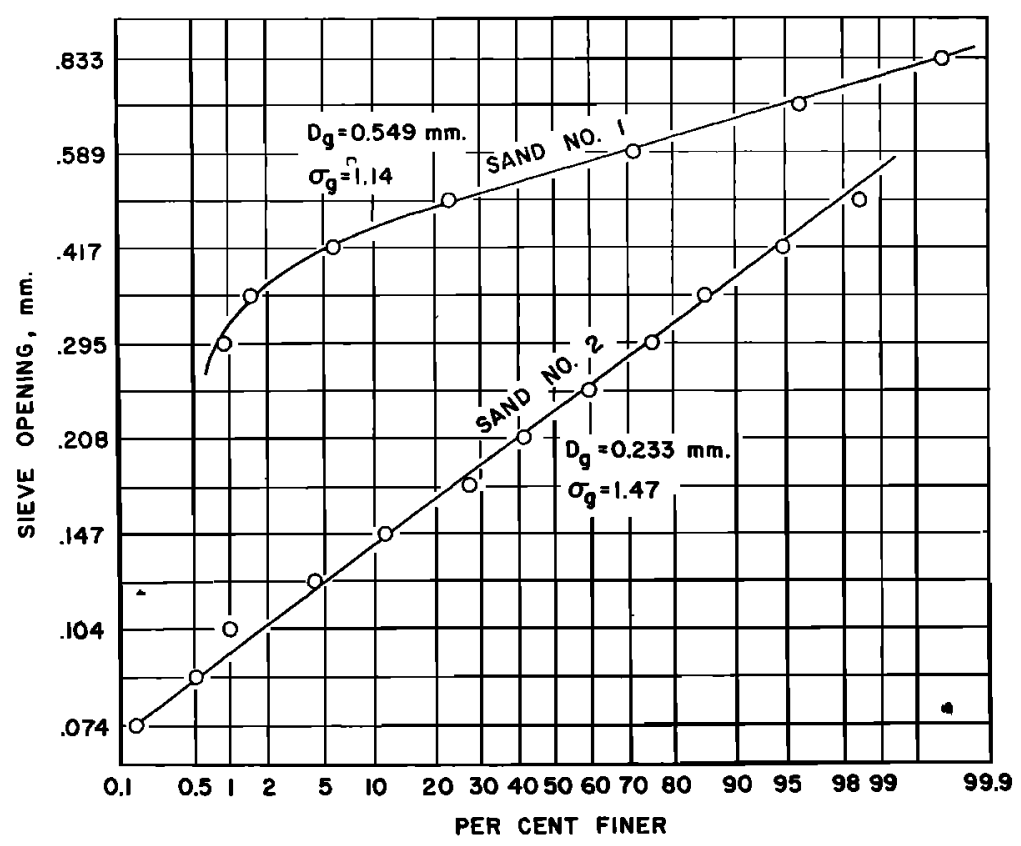

Fig. 5. Cumulative frequency_distributions of sieve diameters.

that the fall velocities of sand 1 can be approximated by either a normal or a log-normal distribution. However, the latter approximation is much better if the part of the curve for velocities less than $w_{15}$ is neglected. It is not surprising that the lower part of the distribution deviates from a log-normal distribution since, as shown in Figure 5, the sieve diameters of the finer 15 per cent of the sediment are not lognormally distributed either. The fall-velocity distribution of sand 2 is very nearly log-normal except for the coarsest 5 per cent of the sediment.

In section 3, it was observed that, if the sieve diameters of a sediment are log-normally distributed, the fall velocities will be expected to be log-normally distributed if the sediment con-

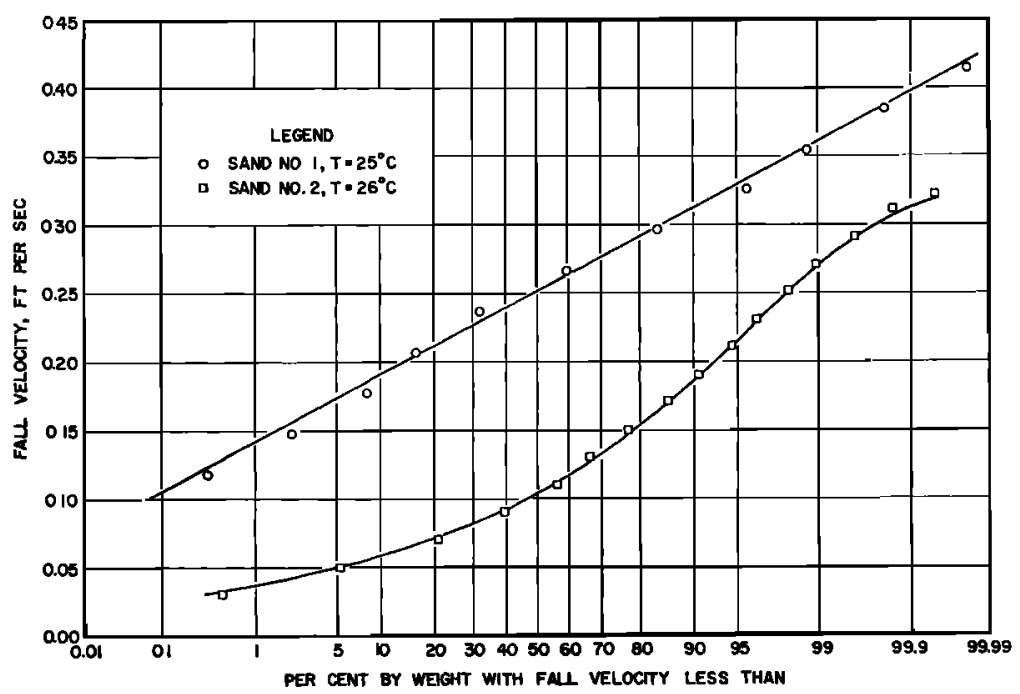

Fig. 6. Arithmetic probability plot of cumulative frequency distributions of fall velocities. 


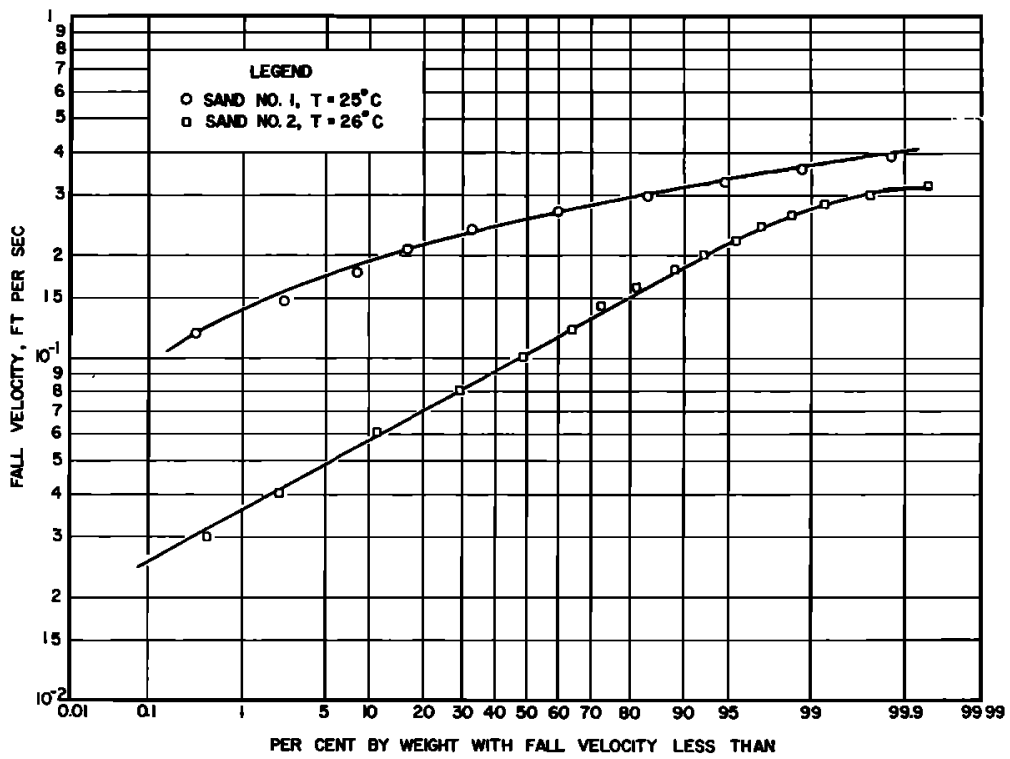

Fig. 7. Logarithmic probability plot of cumulative frequency distributions of fall velocities.

tains a limited range of particle sizes. These two sands tend to corroborate this observation. A further discussion of the relation between the sieve and sedimentation diameters and a summary of the sand characteristics are given in section 6 .

\section{Relation between Sigve Diameters and Sedmantation Diameters}

To determine the mean sedimentation diameter for each sieve fraction, the distribution of fall velocities was first plotted on arithmeticprobability paper. The plotting position $P$ on the abscissa probability scale was that suggested by Thomas [1948],

$$
P=\frac{m}{N+1}
$$

where

$$
\begin{aligned}
m= & \text { rank of particular fall velocity } w(m) \\
& {[m=1,2, \cdots, N ; w(m+1)>w(m)] } \\
P= & \text { percentage of particles with fall velocities } \\
& \text { less than or equal to the corresponding } \\
& \text { value on the ordinate. } \\
N= & \text { number of measurements of fall velocity } \\
& \text { for the sieve fraction. }
\end{aligned}
$$

On a probability plot there was some scatter of the points, but a straight line could be drawn through them quite closely. For the normal distribution represented by this straight line, the mean and the median are the same and could be read directly from the plot. The sedimentation diameter corresponding to this mean diameter was determined from Figure 2. The standard deviation of the fall velocities, $\sigma_{w}$, was also determined from the distribution plot as

$$
\sigma_{w}=w_{50}-w_{15.8}
$$

Table 2 summarizes the data relevant to the distribution of fall velocities for the individual sieve fractions and also gives the sedimentation diameter for each sieve fraction. The last column gives the sedimentation diameter $D_{a_{1}}$ predicted by Figure 8, which was reported by the InterAgency Committee on Water Resources [1957]. The sieve diameter-sedimentation diameter relation given in Figure 8 is for a temperature of $24^{\circ} \mathrm{C}$ and is based on measurements made before 1958 by numerous investigators. The third variable in Figure 8, the shape factor (SF), is the quantity used to define particle shape; it is defined as SF $=c / \sqrt{a b}$, where $a, b, c$ are, respectively, the longest, intermediate, and shortest of three mutually perpendicular axes of the particle. According to the Inter-Agency report, most natural sand particles have a shape factor of about 0.7 , and this value was used to obtain the sedimentation diameters given in the last column of Table 2. The $1^{\circ} \mathrm{C}$ and $2^{\circ} \mathrm{C}$ tem- 
TABLE 2. Summary of Fall-Velocity Measurements of Individual Sieve Fractions

\begin{tabular}{|c|c|c|c|c|c|c|c|}
\hline \multicolumn{2}{|c|}{ Sieve Opening, mm } & \multirow{2}{*}{$\begin{array}{c}\text { Geom. } \\
\text { Mean Sieve } \\
\text { Diameter, } \\
\text { mm }\end{array}$} & \multirow{2}{*}{$\begin{array}{c}\text { No. of } \\
\text { Particles } \\
\text { in } \\
\text { Samples }\end{array}$} & \multirow{2}{*}{$\begin{array}{c}\text { Mean } \\
\text { Fall } \\
\text { Velocity, } \\
\text { ft/sec }\end{array}$} & \multirow{2}{*}{$\begin{array}{c}\text { Std. Dev. } \\
\text { of Fall } \\
\text { Velocity, } \\
\text { ft/sec }\end{array}$} & \multirow{2}{*}{$\begin{array}{c}D_{s} \text { Meas. } \\
\text { Sed. } \\
\text { Diam., } \\
\text { mm }\end{array}$} & \multirow{2}{*}{$\begin{array}{c}D_{s_{1}} \text { Sed. } \\
\text { Diam. from } \\
\text { Fig. 8, } \\
\text { mm }\end{array}$} \\
\hline & & & & & & & \\
\hline
\end{tabular}

\begin{tabular}{|c|c|c|c|c|c|c|c|}
\hline \multicolumn{8}{|c|}{ Sand 1. Temperature $25^{\circ} \pm 1^{\circ} \mathrm{C}$} \\
\hline $0.990^{*}$ & 0.833 & 0.909 & 50 & 0.407 & 0.031 & 0.76 & 0.76 \\
\hline 0.833 & 0.701 & 0.764 & 75 & 0.349 & 0.035 & 0.66 & 0.67 \\
\hline 0.701 & 0.589 & 0.642 & 50 & 0.306 & 0.036 & 0.58 & 0.58 \\
\hline 0.589 & 0.495 & 0.540 & 50 & 0.283 & 0.028 & 0.54 & 0.50 \\
\hline 0.495 & 0.417 & 0.455 & 50 & 0.255 & 0.031 & 0.44 & 0.43 \\
\hline 0.417 & 0.351 & 0.383 & 50 & 0.180 & 0.034 & 0.36 & 0.37 \\
\hline 0.351 & 0.295 & 0.322 & 50 & 0.149 & 0.015 & 0.31 & 0.32 \\
\hline \multicolumn{8}{|c|}{ Sand 2. Temperature $26^{\circ} \pm 1^{\circ} \mathrm{C}$} \\
\hline $0.589^{*}$ & 0.495 & 0.540 & 99 & 0.278 & 0.023 & 0.54 & 0.50 \\
\hline 0.495 & 0.417 & 0.455 & 61 & 0.230 & 0.024 & 0.45 & 0.43 \\
\hline 0.417 & 0.351 & 0.383 & 48 & 0.188 & 0.021 & 0.37 & 0.37 \\
\hline 0.351 & 0.295 & 0.322 & 43 & 0.159 & 0.015 & 0.33 & 0.32 \\
\hline 0.295 & 0.246 & 0.269 & 41 & 0.1305 & 0.0160 & 0.275 & 0.27 \\
\hline 0.246 & 0.208 & 0.226 & 44 & 0.1024 & 0.0120 & 0.230 & 0.23 \\
\hline 0.208 & 0.175 & 0.191 & 54 & 0.0866 & 0.0075 & 0.200 & 0.199 \\
\hline 0.175 & 0.147 & 0.1607 & 33 & 0.0693 & 0.0066 & 0.170 & 0.169 \\
\hline 0.147 & 0.124 & 0.1350 & 60 & 0.0565 & 0.0058 & 0.150 & 0.142 \\
\hline 0.124 & 0.104 & 0.1135 & 60 & 0.0427 & 0.0061 & 0.127 & 0.124 \\
\hline 0.104 & 0.088 & 0.0957 & 60 & 0.0339 & 0.0048 & 0.110 & 0.105 \\
\hline 0.088 & 0.074 & 0.0807 & 57 & 0.0269 & 0.0051 & 0.096 & 0.088 \\
\hline
\end{tabular}

* This sieve was not actually used in the sieve analysis. It is assumed here that all the material would have passed this sieve.

perature discrepancies between the present data and the temperature for Figure $8, T=24^{\circ} \mathrm{C}$, result in differences in the sedimentation diameter of less than 2 per cent. Note that in Figure 8 the relations for the different shape factors can be replaced by lines parallel to the relation for spheres without gross error for fairly wide ranges of the diameters; then over these ranges $D_{\mathrm{s}}$ and $D^{\prime}$ differ by constant factors. This is the approximation that was used to derive $(29 b)$ and to deduce that if the sieve diameters are log- normally distributed the fall velocities will also have this distribution.

In Figure 9 the sedimentation diameters determined in this investigation are compared with the values predicted by Figure 8 . With the exceptions of the fractions retained on the 0.495 $\mathrm{mm}$ and the $0.417-\mathrm{mm}$ sieves and the fraction of sand 2 retained on the $0.074-\mathrm{mm}$ sieve, the agreement with the Inter-Agency values is very good. The discrepancies at the first two of these points are due to the $0.495-\mathrm{mm}$ sieve being

TABLE 3. Summary of Sand Characteristics

\begin{tabular}{|c|c|c|c|c|c|c|c|c|c|}
\hline & $\begin{array}{c}\text { Geom. } \\
\text { Mean } \\
\text { Fall } \\
\text { Velocity } \\
\text { (Fig. 7) } \\
w_{50}, \\
\text { ft/sec }\end{array}$ & $\begin{array}{l}\text { Geom. } \\
\text { Std. Dev. } \\
\text { of Fall } \\
\text { Velocities } \\
\text { (Fig. 7) } \\
\sigma_{w}\end{array}$ & $\begin{array}{c}\text { Geom. } \\
\text { Mean } \\
\text { Sed. } \\
\text { Diam. } \\
\text { (Figs. } 7 \\
\text { and 2) } \\
\left(D_{s}\right)_{50}, \\
\text { mm }\end{array}$ & $\begin{array}{c}\text { Geom. } \\
\text { Mean } \\
\text { Sieve } \\
\text { Diam. } \\
\text { (Fig. 5) } \\
D_{o} \\
\text { mm }\end{array}$ & $\begin{array}{l}\text { Geom. } \\
\text { Std. } \\
\text { Dev. of } \\
\text { Sieve } \\
\text { Diam. } \\
\text { (Fig. 5) } \\
\sigma_{\sigma}\end{array}$ & $\begin{array}{c}\text { Geom. } \\
\text { Mean } \\
\text { Sed. } \\
\text { Diam. } \\
(\text { Fig. 8) } \\
\left(D_{s_{1}}\right)_{50} \text {, } \\
\text { mm }\end{array}$ & 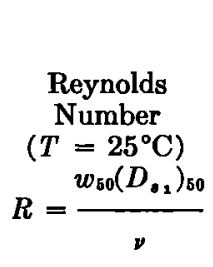 & $\frac{1+m}{2-m}$ & $\begin{array}{c}\text { Com- } \\
\text { puted } \\
\sigma_{w} \\
\text { (Equa- } \\
\text { tion } \\
29 b \text { ) }\end{array}$ \\
\hline Sand 1 & 0.260 & 1.17 & 0.500 & 0.549 & 1.14 & 0.510 & 45.0 & 1.06 & 1.15 \\
\hline Sand 2 & 0.103 & 1.63 & 0.238 & 0.233 & 1.47 & 0.238 & 8.52 & 1.31 & 1.66 \\
\hline
\end{tabular}




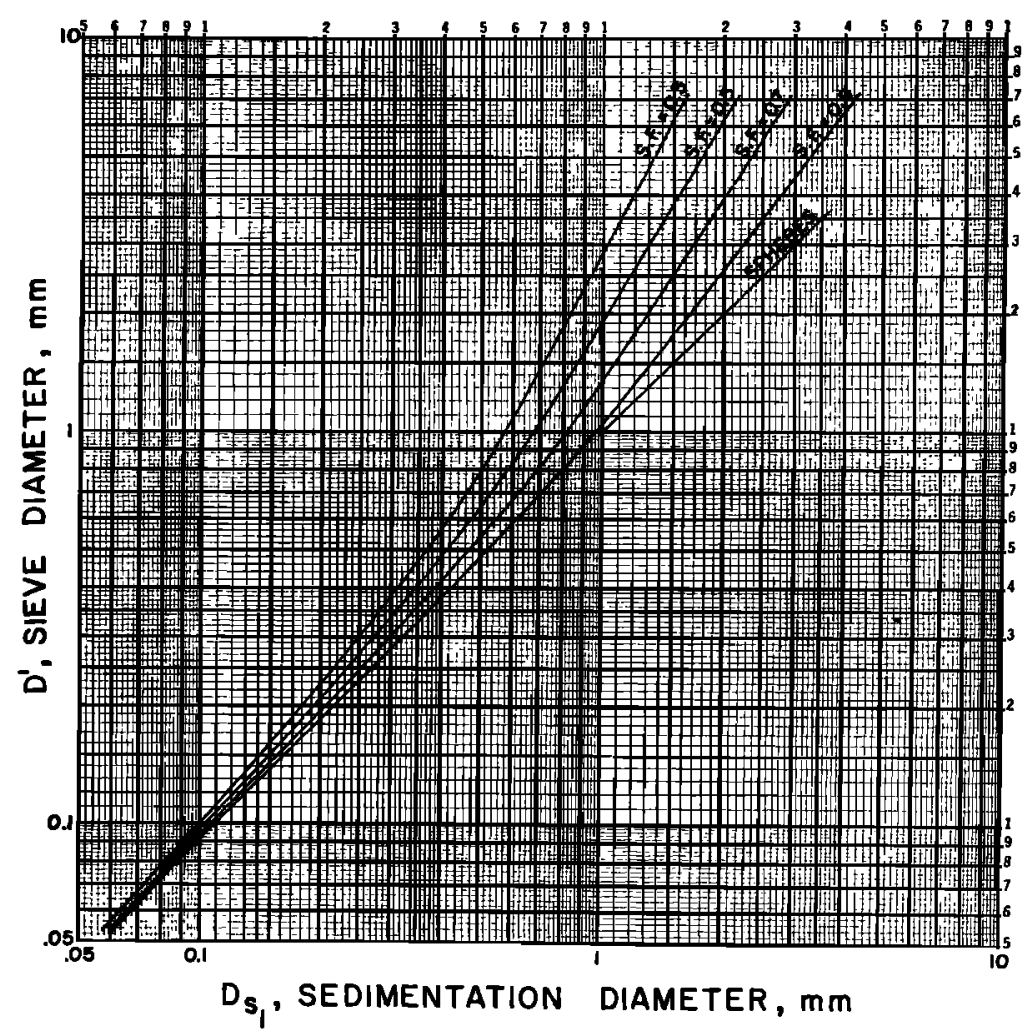

Fig. 8. Relation between sieve diameter and sedimentation diameter. (Taken from Report 12, Inter-Agency Committee on Water Resources, Subcommittee on Sedimentation, 1957.)

faulty. Through use, the sieve had become stretched and the openings had become enlarged. This was discovered after the fall-velocity investigation was completed and the $0.495-\mathrm{mm}$ sieve which had been used was checked against a new sieve. The enlarged openings in the 0.495$\mathrm{mm}$ sieve result in a large sedimentation diameter for the sieve fraction retained on it since the smaller particles were passed to the $0.417-\mathrm{mm}$ sieve. The sieve fraction retained on the 0.417$\mathrm{mm}$ sieve also had a sedimentation diameter which was too large since coarse particles which should have been retained on the $0.495-\mathrm{mm}$ sieve were passed to it. The discrepancy for the sieve fraction of sand 2 retained on the $0.074-\mathrm{mm}$ sieve cannot be explained. However, it is not surprising, since it contained the smallest particles of the sand, and less than $1 / 2$ per cent of the weight of the sand is contained in the sieve fraction. The results of this investigation thus indicate that the relation between sieve diameters and sedimentation diameters given by the Inter-
Agency report for a shape factor of 0.7 is fairly reliable for natural sands.

A summary of the sand characteristics is given in Table 3 . The values of $\left(D_{s_{1}}\right)_{50}$ are the sedimentation diameters corresponding to $D_{a}$ given by Figure 8 . The values of $\left(D_{s_{2}}\right)_{50}$ agree well with the experimentally determined values of the geometric mean sedimentation diameter $\left(D_{a}\right)_{50}$. The computed $\sigma_{v}$ is obtained from (29b) by using the value of $(1+m) /(2-m)$ corresponding to the Reynolds number based on $w_{s 0}$ and $\left(D_{s_{1}}\right)_{50}$. The agreement between the experimentally determined and the computed values of $\sigma_{v}$ is quite good.

Acknowledgments. Equations 7, 8, and 9 were first derived by Professor Norman $\mathrm{H}$. Brooks of California Institute of Technology. Professor Brooks also suggested the analysis that resulted in (27). We gratefully acknowledge these contributions and also his helpful discussion and criticism. We also wish to thank Professor Vito A. Vanoni of Caltech for his many valuable suggestions.

The research reported here was supported by 


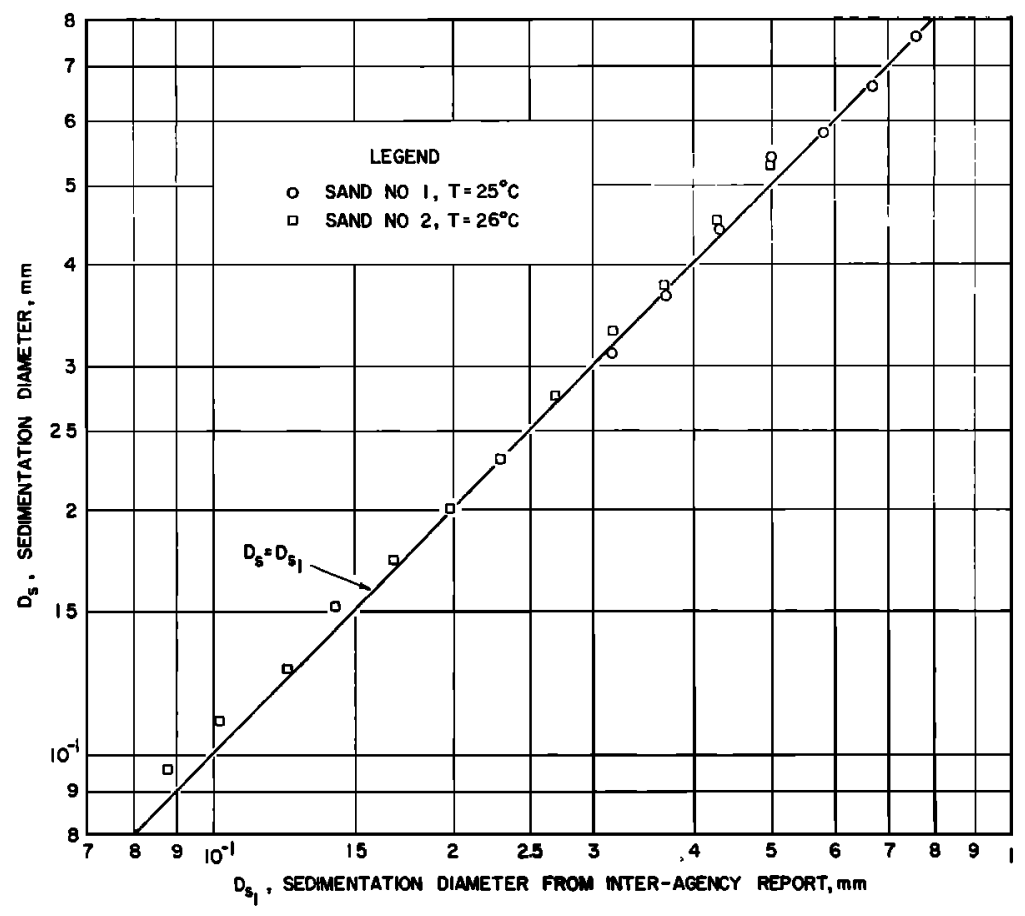

Fig. 9. Comparison of sedimentation diameters determined in this investigation and those given by the Inter-Agency Report.

funds provided by the Agricultural Research Service of the U. S. Department of Agriculture.

\section{REFERENCES}

Kennedy, John F., Stationary waves and antidunes in alluvial channels, Rept. $K H-R-2 .$, W. M. Keck Laboratory of Hydraulics and Water Resources, California Institute of Technology, Pasadena, California, 1961.

Rouse, H. (editor), Engineering Hydraulics, John Wiley \& Sons, New York, 1950.

Otto, George H., A modified logarithmic proba- bility graph for the interpretation of mechanical analysis of sediments, J. Sediment. Petrol., $9(2)$, 329-331, 1939.

Thomas, H. A., Jr., Frequency of minor floods, J. Boston Soc. Civil Engrs., 35, 425-442, 1948.

U. S. Inter-Agency Committee on Water Resources, Subcommittee on Sedimentation, 1957, Some fundamentals of particle size analysis, Rept. 18, U. S. Government Printing Office, 1957.

(Manuscript received August 28, 1961; revised October 4, 1961.) 